\title{
Control of Ferrites Microwave Properties at the French Atomic Energy Commission - Manufacturing Process and Characterization
}

\author{
N. Bardy, G. Leblevennec, D. Le Potier* and J. Authesserre* \\ Commissariat à l'Énergie Atomique, Centre d'Études Scientifiques et Techniques d'Aquitaine, BP. 2, \\ 33114 Le Barp, France \\ * Commissariat à l'Énergie Atomique, Centre d'Études du Ripault, BP. 16, 37260 Monts, France
}

\begin{abstract}
Depending on the desired performances of ferrites, their microwave characteristics must be optimized. Ferrite elaboration relies on highly reproducible compositions and microstructures. In order to fulfil these requirements, manufacturers have to control all the different processing parameters. The selection of the compositional gradation as well as the manufacturing procedure, require accurate and reliable microwave measurement data. A special process has therefore been developed for complex permittivity and permeability measurements. Closely related compositions of ferrites can thus be distinguished and experimental data can be interpreted on the basis of theoretical models.
\end{abstract}

\section{MANUFACTURING PROCESS}

It begins with a careful selection of oxide powders which are mixed by ball milling. At this step, iron contamination through wear of steel balls, is carefully controlled because stoichiometry is a key factor in making the appropriate material. The slip is then atomized in order to get particles ready for synthesis. By calcination, the spinel phase is developed. The synthesis is only partially achieved in order to get a suitable powder for sintering. Due to an efficient grinding, the calcination leads to a powder whose granularity complies with the consolidation process. Powder characterization is carried out by chemical analysis, particle size measurement, morphological observation and specific surface measurement. The latter is carried out by a gas adsorption technique at liquid nitrogen temperature.

Consolidation is achieved by cold isostatic pressing and sintering. A deformable die is filled with ferrite powder and 'sealed. Under 150MPa oil pressure the compaction leads to a roughshape which is ready for the next processing step, after sheath removing. Due to the efficiency of the binder, the green compact can be machined to get, for example, several parts from a large roughshape, or in order to make sure that the right surface will be in contact with the sintering support. Owing to powder characteristics and shape of the die, the cold pressed green compact density is about $2,8 \mathrm{~g} / \mathrm{cm}^{3}$. Densification sintering in air (for NiZn ferrite), is carried out in special furnaces designed by French Atomic Energy Commission engineers in order to solve problems due to the large size of the components and to thermal gradients. Temperature increases slowly up to 1200 or $1300^{\circ} \mathrm{C}$ and decreases also slowly after the flat. Typical sintering cycle needs a week. This is a key factor in order to deal with the 20 per cent linear shrinkage and to generate the correct microstructure related to $\varepsilon$ and $\mu$ properties through control of grain growth. The sintered part is machined by turning and milling with diamond tools under conditions adapted to brittle and rather stiff ferrite material, leading to the final shape of the component.

\section{CONTROL OF RADIOELECTRIC CHARACTERISTICS}

Depending on the intended application, radioelectrical characteristics of the ferrite have to be optimized; once the chosen composition is manufactured, its reproducibility must be controlled. For these reasons, we have developed a procedure to measure complex dielectric permittivity ( $(\varepsilon)$ and magnetic permeability $(\mu)$. We use a classical method suggested by Nicolson and Ross [1]: the material is inserted in a 50 Ohms circular coaxial air line and, from $S$ parameters measured with a network analyser, we compute $\varepsilon$ and $\mu$. The main problem with this method comes from the air gaps existing between the waveguide and the material: they can generate other modes beside the fundamental one. To use the analytical expressions properly, the fundamental mode must be the only propagating one. We therefore have developed a special mounting of the sample in order to eliminate air gaps. The sample has exactly the dimensions of the cross-section of the coaxial line and is kept in position on a brass holder with a low melting point temperature solder composed of $\mathrm{In}, \mathrm{Ga}$, Sn. It can be used for different types of materials 
because it does not penetrate easily, and consequently, material properties are not modified. Figure 1 shows results obtained with and without the special setting; the material under investigation is a ceramic with a real permittivity close to 30 . Without solder, the permittivity is greatly undervalued. This mounting needs a very precise tooling of the sample and holder: dimensions, surface flatness and parallelism. The procedure also works for temperature-dependent characterization and has been qualified from $-80^{\circ} \mathrm{C}$ up to $350^{\circ} \mathrm{C}$.

To validate the method, we have compared results obtained on different materials using this procedure, with other measuring techniques such as resonators, cavities and coaxial discontinuity. A round robin test organized by a french working group under DGA/DRET contract [2], has recently designated this procedure as being the most accurate for the characterization of homogeneous, isotropic, lossy materials. In that case, the accuracy obtained from $50 \mathrm{MHz}$ up to $18 \mathrm{GHz}$ is given on figure 2 .

Figure 1

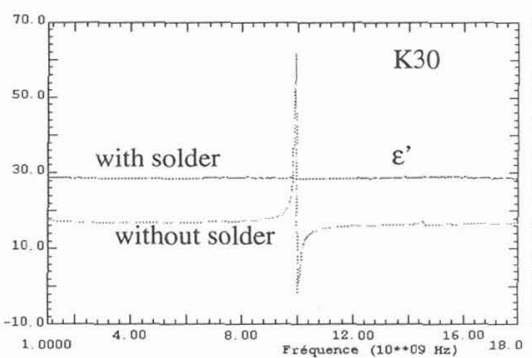

Figure 2

\begin{tabular}{|c|c|c|c|c|}
\hline $\begin{array}{c}\text { Magnitude of } \\
\text { characteristics } \\
\text { values }\end{array}$ & $\mu<0$ & $0<\varepsilon$ or $\mu<1$ & $\begin{array}{c}1<\varepsilon \text { or } \mu< \\
\text { several units }\end{array}$ & $\begin{array}{c}\varepsilon \text { or } \mu> \\
\text { several units }\end{array}$ \\
\hline$\varepsilon^{\prime}$ & - & - & $+1-0.05$ & \multirow{2}{*}{$+1-3 \%$} \\
\hline$\varepsilon^{\prime \prime}$ & - & $+1-0.2$ & \\
\hline$\mu^{\prime}$ & & $+1-0.05$ & \\
\hline$\mu^{\prime \prime}$ & - & & $+1-0.05$ \\
\hline
\end{tabular}

When applied to the characterization of ferrites, this method gives the dielectric and magnetic broadband spectra. Closely related compositions of ferrites can be distinguished by means of their permeability values as shown in figures 3 and 4 . The fundamental properties of the material contained in such results range from static properties such as saturation magnetization or anisotropy constant, to dynamic properties such as relaxation phenomena.

For example, an accurate way to get the value of the saturation magnetization Is of the ferrite is to pick up the frequency point where the imaginary part of the magnetic permeability reaches zero at high frequency. This frequency $f(\mu$ " $=0)$ is directly related to the magnetization through the gyromagnetic ratio $\gamma \cdot 2 \pi \mathrm{f}=\gamma \mathrm{is}$.

The magnetic permeability spectrum exhibits two typical magnetic dispersions. At low frequencies, near $3 \mathrm{MHz}$, with a rather large amplitude dispersion, we find the domain wall relaxation. At higher frequencies, around $30 \mathrm{MHz}$, one can distinguish on the curves the natural spin resonance (N.S.R) of the polycristal. These two contributions can be splitted using numerical techniques to decompose the whole spectrum into Debye functions which can then be further analysed in terms of the specific mechanism and the specific characteristics of each relaxation.

Although only the $\mu$-curves are given below, it is worth mentioning that the dielectric properties are very sensitive and well related to the anionic vacancies and to the grain boundary quality resulting from the thermic cycle used during sintering. All these measurements are of prime importance for the choice of the composition of ferrites and elaboration process control.
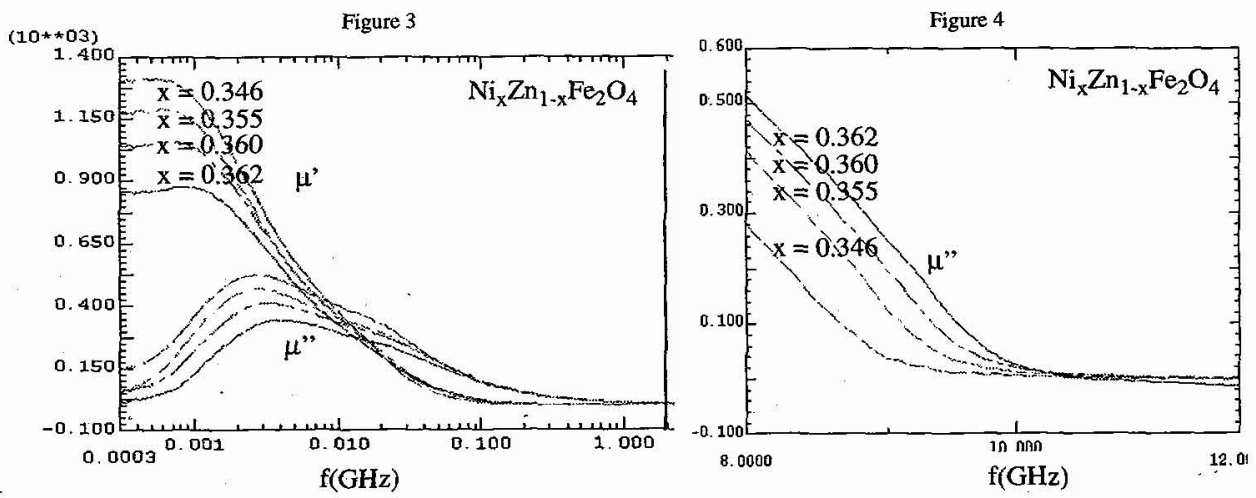

\section{References}

(1) A. M. Nicolson, G. F. Ross «Measurement of the intrinsic properties of materials by time domain techniques» IEEE Trans. Instrum. Meas. vol MM-17, pp395-402, Dec 1968

(2) "Caractérisation Microonde des Materiaux» Convention de Recherche DRET 92/117/A 